\title{
Zumkeller Labeling of Complete Graphs
}

\author{
B. J. Balamurugan, R. Madura Meenakshi
}

Abstract: Let $G(V, E)$ be a graph with vertex set $V$ and edge set $E$. The process of assigning natural numbers to the vertices of $G$ such that the product of the numbers of adjacent vertices of $G$ is a Zumkeller number on the edges of $G$ is known as Zumkeller labeling of $G$. This can be achieved by defining an appropriate vertex function of $G$. In this article, we show the existence of this labeling to complete graph and fan graph.

Keywords: Zumkeller Number, Labeling, Injective Function and Complete Graph, Fan Graph.

\section{INTRODUCTION}

In the theory of graph labeling, the labels are computed to vertices or edges of the graph through mathematical functions. In the year 1960, Alex Rosa[13] had introduced first the graph labeling concept. For the recent research trends in graph labeling and history of graph labeling one can refer [7]. The notations and terminology of graph theory used in this paper are referred to Harary [9]. The applications of labelled graphs have found in recent research.

The applications of graceful labeling of complete graph to coding theory are presented in [8]. Krishnappa H.K et.al in $[11,12]$ showed the practical applications of the complete graph $K_{n}$ in the field of cryptography. Zongheng et al. [15] discussed the connectivity and coverage problems in sensor network. Various algorithms were presented for the selection of minimum number of nodes for a communication graph which results in different graph topologies. Jonathann et al. [10] explored the applications of graph labeling in network coding related to mobile communications.

The Zumkeller graphs were introduced by Balamurugan et al in [3] in the year 2014. Also in the literature [1-6],the Zumkeller graphs, Strongly multiplicative Zumkeller graphs and k-Zumkeller graphs have been introduced and investigated by Balamurugan et al. In this paper, we investigate the existence of Zumkeller complete graphs and Zumkeller fan graphs.

\section{PRELIMINARIES}

We refer the paper [14] for the concept and properties of Zumkeller numbers.

\section{Definition 2. 1.}

A Zumkeller number $\mathrm{n}$ is a real positive integer in which the positive factors of $\mathrm{n}$ can be partitioned into two disjoint sets

Revised Manuscript Received on December 16, 2019.

* Correspondence Author

B.J.Balamurugan*, School of Advanced Sciences, VIT University, Chennai Campus, Chennai-600127, Tamil Nadu, India. Email: balamurugan.bj@vit.ac.in

R. Madura Meenakshi, Research Scholar, SSN College of Engineering, Kalavakkam-603110, Tamil Nadu, India. Email: madurameenakshir@ssn.edu.in
Such that the sum of the integers in the first set is equal to the sum of the integers in the second set.

This partition of $\mathrm{n}$ is known as Zumkeller partition.

\section{Example 2.1}

40 is a Zumkeller number because the positive factors of 40 can be partitioned into $A=\{5,40\}$ and $B=\{1,2,4,8,10,20\}$ where the sum of each set is 45 .

6 is the first Zumkeller number and other few Zumkeller numbers are $12,20,24,28,30, \ldots$.whose partitions are as follows:

$\begin{array}{cll}6 & {[6]} & {[1,2,3]} \\ 12 & {[2,12]} & {[1,3,4,6]} \\ 20 & {[1,20]} & {[2,4,5,10]} \\ 24 & {[6,24]} & {[1,2,3,4,8,12]} \\ 28 & {[28]} & {[1,2,4,7,14]} \\ 30 & {[6,30]} & {[1,2,3,5,10,15]}\end{array}$

\section{Remark}

The numbers which are perfect square or twice the perfect square are not Zumkeller numbers.

\section{MAIN RESULTS}

In this section, we prove the complete graph $\mathrm{K}_{\mathrm{n}}$, one point union of two complete graphs $K_{n}^{(2)}$ and fan graph $F_{n}=P_{n}+K_{1}$ admit the Zumkeller labeling.

\section{Definition3.1}

A simple graph $G=(V, E)$, where $\mathrm{V}$ is vertex set and $\mathrm{E}$ is edge set of $\mathrm{G}$, is said to admit a Zumkeller labeling if there exists an injective function $f: V \rightarrow N$ such that the induced edge function $f^{*}: E \rightarrow N$ defined as $f^{*}(x y)=f(x) f(y)$ is a Zumkeller number for $x y \in E$; $x, y \in V$. The labelled graph $\mathrm{G}$ is called as a Zumkeller graph.

\section{Example 3.1}

The graph shown in the Fig.2.1 is a Zumkeller graph.

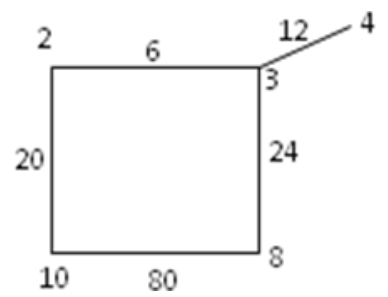

Fig.2.1: A Zumkeller Graph 


\section{Definition3.2}

A simple graph in which every pair of vertices adjacent is called a complete graph.

\section{Theorem 3.1:}

The complete graph $K_{n}$ is a Zumkeller graph when $n \equiv 2(\bmod 4)$ and $n \leq 10$.

\section{Proof:}

Let $V=\left\{\mathrm{v}_{\mathrm{i}} \mid 1 \leq \mathrm{i} \leq \mathrm{n}\right\}$ be the vertex set and $E=\left\{e_{i j} \mid 1 \leq i \leq n, 1 \leq j \leq n\right.$ and $\left.i \leq j\right\}$ be the edge set of the complete graph $K_{n}$.

Define an injective function $f: V \rightarrow N$ such that

$$
\begin{aligned}
& f\left(v_{1}\right)=\frac{n}{2}\left(\frac{n}{2}+1\right) \\
& f\left(v_{i}\right)=\left(\frac{n}{2}+i-1\right)\left(\frac{n}{2}+i\right) \text { for } 2 \leq i \leq n
\end{aligned}
$$

Define an induced function $f^{*}: E \rightarrow N$ such that $f^{*}\left(e_{i j}\right)=f^{*}\left(v_{i} v_{j}\right)=f\left(v_{i}\right) f\left(v_{j}\right)$.

Let $f\left(v_{i}\right)=a \times b$ and $f\left(v_{j}\right)=c \times d$ where $a, b$ and $c, d$ are a pair of consecutive integers.

Claim: To prove $f\left(v_{i}\right) f\left(v_{j}\right)=$ abcd is a Zumkeller number.

Now,

Since $n \leq 10$,

$$
f\left(v_{n}\right)=\left(\frac{n}{2}+n-1\right)\left(\frac{n}{2}+n\right)
$$

We have $f\left(v_{n}\right) \leq 14 \times 15$

Here the integers in the product of two consecutive integers are less than or equal to 15 . All odd numbers other than 9 and 15 are primes in this product and in each pair of consecutive numbers there is an odd number.

Therefore, the product abcd of those numbers have the prime factorisation of the form $2^{k} p_{1}^{k_{1}} p_{2}^{k_{2}} \ldots p_{m}^{k_{m}}$ for which at least one $k_{i}$ is odd.

Now we prove that the products of any two numbers in the set $\{8 \times 9,9 \times 10,14 \times 15\}$ are also the same form of prime factorisation.

$$
\begin{aligned}
& \text { (i) } 8 \times 9 \times 9 \times 10=2^{4} 3^{4} 5, \\
& \text { (ii) } 8 \times 9 \times 14 \times 15=2^{4} 3^{3} 5 \\
& \text { (iii) } 9 \times 10 \times 14 \times 15=2^{2} 3^{3} 57
\end{aligned}
$$

Therefore, all the edges in the complete graph $\mathrm{K}_{\mathrm{n}}$ have the prime factorisation of the form $2^{k} p_{1}^{k_{1}} p_{2}^{k_{2}} \ldots p_{m}^{k_{m}}$ in which at least one $k_{i}$ is odd.

By the property of Zumkeller numbers, abcd is a Zumkeller number.

Hence the complete graph $\mathrm{K}_{\mathrm{n}}$ admits a Zumkeller labeling when $n \equiv 2(\bmod 4)$ and $n \leq 10$.

An illustration of this theorem is shown in the example 3.1.

\section{Example 3.2}

The graph shown in the Fig.3.1 is a Zumkeller labeling of $K_{6}$

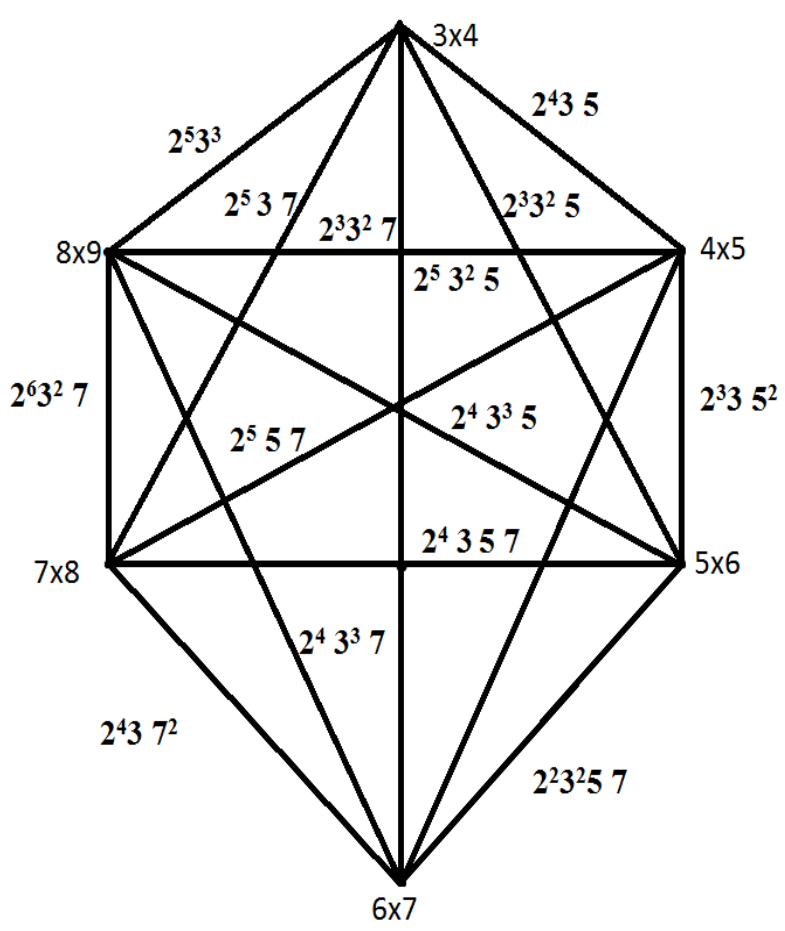

Fig.3.1. Zumkeller labelling of $K_{6}$

\section{Definition 3.3}

The one-point union of two complete graphs is a graph $K_{n}^{(2)}(n \geq 3)$ consists of two copies of $K_{n}$ sharing a common vertex. It contains $2 n-1$ vertices and $n(n-1)$ edges.

\section{Theroem3.2:}

The graph $K_{n}^{(2)}$ admits a Zumkeller labeling when $n \leq 6$.

\section{Proof:}

Let $V=\left\{v_{i} \mid 1 \leq i \leq n-1\right\} \cup\left\{u_{i} \mid 1 \leq i \leq n-1\right\} \cup\left\{v_{0}\right\}$

be the vertex set and $E=\left\{e_{i j} \mid 1 \leq i \leq n-1,1 \leq j \leq n-1\right.$ and $\left.i<j\right\}$

$\cup\left\{e_{i j}^{\prime} \mid 1 \leq i \leq n-1,1 \leq j \leq n-1\right.$ and $\left.i<j\right\}$

$\cup\left\{e_{0 i} \mid 1 \leq i \leq n-1\right\} \cup\left\{e_{0 j} \mid 1 \leq j \leq n-1\right\}$

be the edge set of the graph $K_{n}^{(2)}$.

Define an injective function $f: V \rightarrow N$ such that

$$
\begin{aligned}
& f\left(v_{0}\right)=8 \times 9 \\
& f\left(v_{i}\right)=(i+2) \times(i+3) \\
& f\left(u_{i}\right)=(i+8) \times(i+9)
\end{aligned}
$$

Define an induced function $f^{*}: E \rightarrow N$ such that

$$
\begin{gathered}
f^{*}\left(e_{i j}\right)=f^{*}\left(v_{i} v_{j}\right)=f\left(v_{i}\right) f\left(v_{j}\right) \\
f^{*}\left(e_{i j}^{\prime}\right)=f^{*}\left(u_{i} u_{j}\right)=f\left(u_{i}\right) f\left(u_{j}\right) \\
f^{*}\left(e_{0 i}\right)=f^{*}\left(v_{0} v_{i}\right)=f\left(v_{0}\right) f\left(v_{i}\right)=8 \times 9 f\left(v_{i}\right) \\
f^{*}\left(e_{0 j}\right)=f^{*}\left(v_{0} v_{j}\right)=f\left(v_{0}\right) f\left(v_{j}\right)=8 \times 9 f\left(v_{j}\right)
\end{gathered}
$$

All the edges receive the Zumkeller numbers. This can be proved as in Theorem3.1. 
Hence The graph $K_{n}^{(2)}$ admits a Zumkeller labeling when $n \leq 6$.

Theorem 3.2 is illustrated in the following example 3.2.

\section{Example 3.3}

The Fig.3.2 shows the existence of the Zumkeller labeling for the graph $K_{6}^{(2)}$.

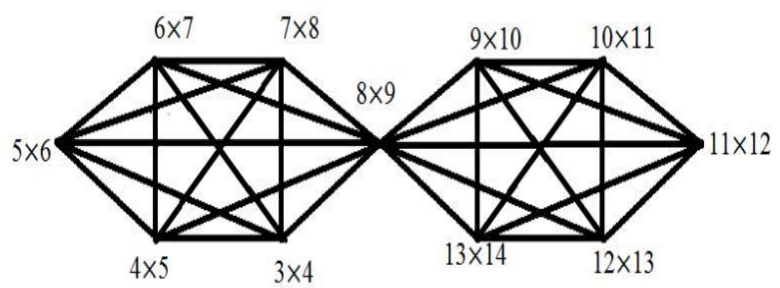

Fig.3.2. $\quad K_{6}^{(2)}$ is a Zumkeller graph

\section{Theorem 3.3}

The fan graph $F_{n}=P_{n}+K_{1}$ is a Zumkeller graph.

\section{Proof:}

Let $V=\left\{v_{0}, v_{1}, v_{2}, \ldots, v_{n}, v_{n+1}\right\}$ be the vertex set of the fan graph $F_{n}$, where $v_{0}$ is the apex vertex.

Let

$E=\left\{e_{i}=v_{i} v_{i+1} \mid 1 \leq i \leq n\right\} \cup\left\{e_{i}^{\prime}=v_{0} v_{i} \mid 1 \leq i \leq n+1\right\}$

be the edge set of the fan graph.

Define an injective mapping $f: V \rightarrow N$ such that

$f\left(v_{i}\right)=2^{\frac{i+1}{2}}$,

$f\left(v_{i+1}\right)=p_{1} 2^{\frac{i+1}{2}}, i=1,3,5 \ldots n$

$f\left(v_{0}\right)=2 p_{2}$ where $p_{1}, p_{2}$ are distinct prime numbers

which are greater than 2 but less than 10 and an induced function $f^{*}: E \rightarrow N$ such that

$f^{*}\left(e_{i}\right)=f^{*}\left(v_{i} v_{i+1}\right)=f\left(v_{i}\right) f\left(v_{i+1}\right), \quad \mathbf{1} \leq \boldsymbol{i} \leq \boldsymbol{n}$

$f^{*}\left(e_{i}^{\prime}\right)=f^{*}\left(v_{0} v_{i}\right)=f\left(v_{0}\right) f\left(v_{i}\right), \quad 1 \leq i \leq n+1$

Now we claim that the numbers on the edges are Zumkeller numbers.

$$
\begin{aligned}
f^{*}\left(e_{i}\right) & =f^{*}\left(v_{i} v_{i+1}\right)=f\left(v_{i}\right) f\left(v_{i+1}\right) \\
& =2^{\frac{i+1}{2}} p_{1} 2^{\frac{i+1}{2}}=p_{1} 2^{i+1}
\end{aligned}
$$

is a Zumkeller number and when $i \equiv 1(\bmod 2)$

(i) $f^{*}\left(e_{i}^{i}\right)=2 p_{2} 2^{\frac{i+1}{2}}=p_{2} 2^{\frac{i+3}{2}}$.

(ii) $f^{*}\left(e_{i+1}^{\prime}\right)=2 p_{1} p_{2} 2^{\frac{i+1}{2}}=p_{1} p_{2} 2^{\frac{i+3}{2}}$

are Zumkeller numbers.

Hence the fan graph $F_{n}$ is a Zumkeller graph.

Example 3.3

The fan graph $F_{6}$ in Fig 3.3 is a Zumkeller graph when $p_{1}=3, p_{2}=5$

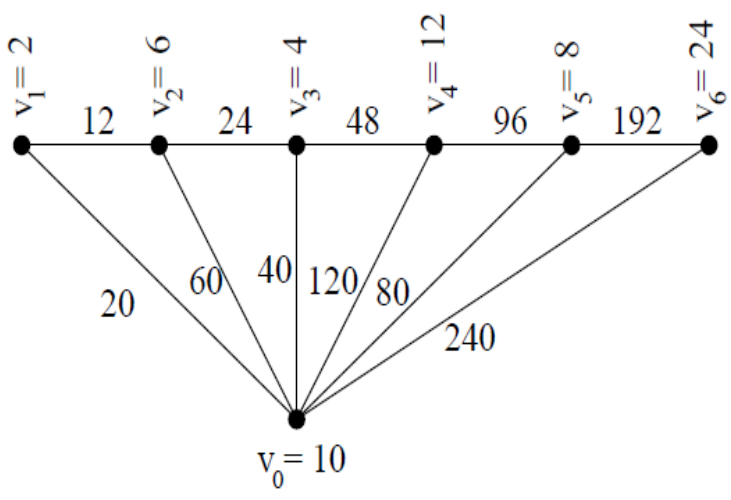

Fig 3.3. $F_{6}$ is a Zumkeller graph

\section{CONCLUSION}

In this research article, the existence of the Zumkeller labeling to the following graphs

(i) The complete graph $K_{n}$, when $n \leq 10$

(ii) The one-point union of two complete graphs when $n \leq 6$.

(iii) The fan graph $F_{n}=P_{n}+K_{1}$

are proved.

Finding the other classes of graphs which will admit the Zumkeller labeling is a future scope of this research area.

\section{REFERENCES}

1. Balamurugan, B.J., Thirusangu, K., Thomas, D.G.: Strongly multiplicative Zumkeller labeling of graphs. In International Conference on Information and Mathematical Sciences. Elsevier (2013) 349-354.

2. Balamurugan, B.J., Thirusangu, K., Thomas, D.G.: Strongly multiplicative Zumkeller labeling for acyclic graphs. Proceedings of International Conference on Emerging Trends in Science, Engineering, Business and Disaster Management (ICBDM - 2014). to appear in IEEE Digital Library.

3. Balamurugan, B.J., Thirusangu, K., Thomas, D.G.: Zumkeller labeling of some cycle related graphs. Proceedings of International Conferenc on Mathematical Sciences (ICMS - 2014). Elsevier (2014) 549-553.

4. Balamurugan, B.J., Thirusangu, K., Thomas, D.G.: Zumkeller labeling algorithms for complete bipartite graphs and wheel graphs. Advances in Intelligent Systems and Computing. Springer. 324 (2015) 405-413.

5. Balamurugan, B.J., Thirusangu, K., Thomas, D.G.: Algorithms for Zumkeller labeling of full binary trees and square grids. Advances in Intelligent Systems and Computing, Springer. 325 (2015) 183-192.

6. Balamurugan, B.J., Thirusangu, K., Thomas, D.G.: k-Zumkeller labeling for twig graphs. Electronic Notes in Discrete Mathematics, Elsevier, 48(2015) $119-126$.

7. Gallian, J.A.: A dynamic survey of graph labeling. Electronic Journal of Combinatorics. 17 (DS6) (2013).

8. Gary S. Bloom and Solomon W.Golomb.: Applications of numbered undirected graphs. Proceedings of the IEEE, Vol. 65, No.4, (April 1977) 562-570.

9. Harary, F.: Graph theory. Addison-Wesley. Reading Mass (1972).

10. Jonathann Webb, Fernando Docemmilli, Makhailn Bonin,: Graph Theeory Applications in network Security. Cyber Security \& Digital Forencis Workshops, Australia (September 2015),

11. Krishnnappa H. K, N.K.Srinath and P.Ramakanth Kumar, Vertex Magic Total Labelings of complete graphs, IJCMSA., Vol 4, No 1-2(2010) 157-169

12. Krishnnappa H. K, N.K.Srinath and P.Ramakanth Kumar, Vertex Magic Total Labelings of complete graphs and their application for public-key cryptosystem, IJIRCCE, Vol. 1, Issue 2, (April 2013) 379-387 
13. Rosa, A.: On certain valuations of the vertices of a graph. In N.B. Gordan and Dunad, editors, Theory of graphs. International Symposium. Paris (1966) 349-359.

14. Yuejian Peng, Bhaskara Rao, K.P.S.: On Zumkeller numbers. Journal of Number Theory. 133(4) (2013) 1135-1155.

15. Zongheng Zhou, S. Das and H. Gupta.: Connected k-coverage problem in sensor networks, Proceedings. $13^{\text {th }}$ International Conference on Computer Communications and Networks (IEEE Cat. No.04EX969), Chicago, IL, (2004) 373-378

\section{AUTHORS PROFILE}

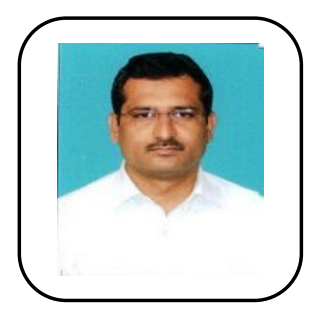

Dr.B.J.Balamurugan, received his Ph.D degree in Mathematics from the University of Madras, Chennai, India. Currently, he is an Assistant Professor(Senior) of Mathematics in the School of Advanced Sciences at VIT University, Chennai Campus, Chennai, India. He has more than 22 years of teaching experience at Undergraduate and Postgraduate level courses. Dr.B.J.Balamurugan has published more than 32 research papers in various journals and conference proceedings. His research interest includes graph theory, graph grammars, fuzzy logic and Petri nets.

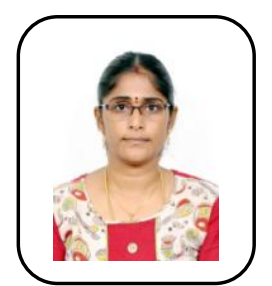

R. Madura Meenakshi received her M.Sc and M.Phil degrees in Mathematics from Avinashilingam University, Coimbatore, Tamil Nadu, India. Currently, she is a research scholar at SSN College of Engineering, Chennai, India. Her research interest includes Graph Theory, Image Processing and Machine Learning. 